\title{
A SURVEY ON CLUSTER BASED ENERGY EFFICIENT ROUTING PROTOCOLS
}

\begin{tabular}{|l|l|l|}
\hline Shwetha.M (M.Tech) & Shashikala(Ph.D) & Dr.Kavitha.C, Ph.D \\
B.N.M. Institute of Technology, & Associate professor & Professor \\
Bangalore, Karnataka, India. & B.N.M. Institute of Technology, & B.N.M. Institute of Technology, \\
Email: km.shwetha50@gmail.com & Bangalore, Karnataka, India. & Bangalore, Karnataka, India. \\
& Email: hebbar.Shahikala@gmail.com & Email: Kavitha_Prasanna@yahoo.com \\
\hline
\end{tabular}

\begin{abstract}
Wireless sensor networks (WSNs) have been mainly deployed in remote areas for usually environment monitoring purposes, military application, infrastructure monitoring etc. It is very difficult to maintain or repair sensor nodes every now and then in these environment. Moreover, sensor nodes have limited resources. Hence it's very necessary to find an approach that is very effective in routing data among the nodes and preserve the lifetime of the network. So in the course of time this document presents a survey on clustering technique designed to preserve energy as well as route in an energy efficient manner within wireless sensor networks. Before routing is done, a clustering algorithm is applied to the node locations to aggregate nodes into cluster whose diameter is comparable to the node communication radius. Each cluster elects a cluster-head node, and routing is done only among the cluster-heads. This is advantageous for a variety of reasons, including the possibility of using simpler communication protocols within a cluster, recycling of resources among disjoint clusters, saving power and reducing the number of nodes from participating in routing. All these advantage will lead to distribute the load evenly among the nodes in network and thereby avoiding drainage of energy of any node that becomes the hot-spot while routing.
\end{abstract}

\section{INTRODUCTION}

\section{Wireless Sensor Networks (WSNs)}

WSN is a collection of hundreds and thousands of low cost, low power smart sensing devices. In most cases, the tiny sensor nodes are equipped with a radio transceiver, an antenna, a processor, memory, and battery. These devices are typically limited in terms of computation and wireless communication capabilities. Sensor nodes are deployed in a monitoring area. They collect data from monitoring environment and transmit to base station (BS) by multi-hope or single hope communication.
BS performs more intricate processing tasks. Basically, a BS acts as a gateway to the outside network (e.g., Internet) or the connecting point for end-user interface. As WSNs are deployed in a vast area, not all sensor nodes can communicate directly with the BS. Wireless communications are used for the collaboration among sensor nodes to transmit data to the BS using a set of predefined rules and instructions called a routing protocol. Routing is a fundamental functionality in WSN in order to forward data packets from source node to the destination node with the support of intermediate nodes.

Sensor nodes are used in a wide variety of applications ranging from household needs to military purposes. Area monitoring is a common application of WSNs. In area monitoring, the WSN is deployed over a region where some phenomenon is to be monitored. For example in military, sensors are used to detect enemy intrusion and in geo-fencing of gas or oil pipelines the civilian is the intruder. A network of Sensor Nodes can be installed in a forest to detect when a fire has started. Also sensors can be used for infrastructure maintenance, inside home appliances like refrigerators, air cooler etc.

There are many routing techniques used in WSNs, but the most efficient among them is Cluster Based Energy Efficient Routing Technique (CBER). This technique considers the energy constraints during routing. Initially a cluster is formed, where cluster head is chosen among other nodes (cluster members).So cluster is group of nodes which are in radio range of each other, involves grouping nodes into clusters and electing a cluster head. The size of the cluster is usually the communication range 
of a node in sensor network. Here cluster head is elected mainly based on minimal residual energy or any other criteria based on the application. Once the cluster is formed, internetwork and intra-network routing takes place. Data aggregated by cluster heads from member nodes within each clusters are routed towards the sink node (Base station). Such routing may take place directly between a cluster head to sink node or between intermediate cluster heads and finally to sink node.

An advantage of clustering involves hierarchical structures to be built on the nodes and enables more efficient use of scarce resources such as frequency spectrum, bandwidth and power. Reuse of resources across non-overlapping clusters along with simple routing protocols within the clusters. Also allows monitoring of the health of network as the nodes in cluster can play the role of watchdogs over other nodes. Comprises of mixture of nodes such as one having more power or special capabilities of increased communication range, GPS and the like. Clustering can be used to thin out parts of the network where an excessive number of nodes may be present. Reduces communication overhead for both single and multi hop and reducing number of nodes taking part in transmission.

\section{RELATED WORKS}

[1] Ankit Thakkar, et al., has proposed a method that takes care of time critical applications like forest fire detection and battle field monitoring which demands reception of data from the source with the bounded delay to avoid disasters. This method enhances the network lifetime and provides information to the sink with a bounded delay. In this proposed approach, a routing algorithm named Energy Delay Index for Trade-off (EDIT) is used to optimize both energy and delay. The EDIT is used to select cluster heads and "next hop" by considering energy and/or delay requirements of a given application. Proposed approach is derived using two different aspects of distances between a node and the sink named Euclidean distance and Hop-count, and further proves using realistic parameters of radio to get data closest to the test bed implementation. The results aspire to give sufficient insights to others before doing test bed implementation.

The proposed algorithm works in rounds and each of these rounds are divided into two phases:

i) Cluster Setup Phase and

ii) Steady State Phase.

A neighbor discovery phase is executed once before the commencement of the first round and it is explained below.

\section{A. Neighbor Discovery Phase}

Algorithm begins with neighbor discovery phase which is initiated by the sink by sending a Hello packet. Receiving nodes of Hello packet add sender as its neighbor and record information like Sender Id, Hop-count and location, and then send Hello Reply to the sender. Whenever any node is having its energy less than threshold (depending on application), it will broadcast itself as a dead node by sending Dead message. The receiving nodes update their neighbor table on reception of Dead messages. Neighbor discovery phase should be done only once at the time of network deployment.

\section{B. Cluster Setup Phase}

Selection of cluster head is based on minimum communication energy expenditure between non- cluster head node and selected cluster head node. After selection of cluster head node, non- cluster head nodes will send cluster head Join message including their current energy level. Each final cluster head node prepares TDMA schedule for its own member nodes from which it has received cluster head JOIN messages. It also selects one of the member nodes as a gateway node if two cluster head nodes are not in a communication range of each other. Selection of a gateway node depends on the energy/delay requirements of the underlying application. 


\section{Steady State Phase}

All nodes remain in sleep state except cluster head nodes. Data transmission from non-cluster head nodes to cluster head node is done as per the TDMA schedule announced by the cluster head. This scheme avoids collision of the data messages, and each member node remains in transmit state for a short duration. This helps to save energy of the member nodes.

[2] Sajid Hussain et al., proposed that cluster based routing technique is an energy efficient routing technique compared to any other technique. Hierarchical cluster-based routing (HCR) technique is an extension of the LEACH protocol that is a self organized cluster-based approach for continuous monitoring. In $\mathrm{LEACH}$, the network is randomly divided into several clusters, where each cluster is managed by a cluster head $(\mathrm{CH})$. The sensor nodes transmit data to their cluster heads, which transmit the aggregated data to the base station. In HCR, each cluster is managed by a set of associates and the energy efficient clusters are retained for a longer period of time; the energy efficient clusters are identified using heuristics-based approach. Moreover, in a variation of HCR, the base station determines the cluster formation.

A Genetic Algorithm (GA) [6] is used to generate energy-efficient hierarchical clusters. The base station broadcasts the GA-based clusters configuration, which is received by the sensor nodes and the network is configured accordingly.GA also known as a global heuristic algorithm.

Manjula R, Student Member, IEEE et al.,[3] proposed an energy efficient privacy preserved routing algorithm where the event (i.e., asset) detected nodes called as source nodes report the events' location information to the Base Station(BS) using phantom source (also known as phantom node) concept and alpha-angle anonymity concept. Routing is done using existing greedy routing protocol. Comparison through simulations shows that the current solution reduces the energy consumption and delay while maintaining the same level of privacy as that of two existing popular techniques. This approach also gives a method to measure the energy consumption and delay in terms of number of hops that a packet takes to reach the base station.

After an event is detected, a nearby node becomes the source that prepares an event (or asset) detect information packet to be routed to the BS using a shortest path routing (SPR) algorithms as shown in Fig.2.1 (a). The adversary who attacks the network, positions itself one or two hops away from the BS so that it can access all the packets arriving at the BS and trace back to the source node for gaining control of the asset. From Fig.2.1 (a) it is clear that the adversary will reach the source in a short interval of time if no privacy protection is used. Random walk routing (RWR) mechanism called phantom routing is used to achieve source location privacy. In these protocols once a node that detects an event prepares a packet having the event detected information and sends it to the BS. Initially this packet undergoes a random walk for few hops from the source and thereafter the packet is sent to the BS using a shortest path routing as shown in Fig2.1 (b). The premise of this approach is that even if an adversary is able to track back the routed path (i.e., path taken by the packet to reach the BS), it would only be able to figure out the terminal node (i.e., phantom source) of the random walk instead of the original data source. The benefit of using RWR is that the adversary needs more time to reach the asset when compared to the time taken by adversary to reach the asset if SPR were used. This difference can be observed in Fig. 2.1(b). This gives the asset (panda in this case) extra safety period to move away from its current position before the adversary arrives. The safety period of a routing protocol for a given adversarial movement strategy is the number of messages initiated by the source node that is monitoring an asset. 


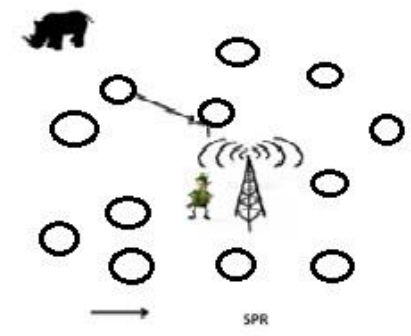

(a) SPR

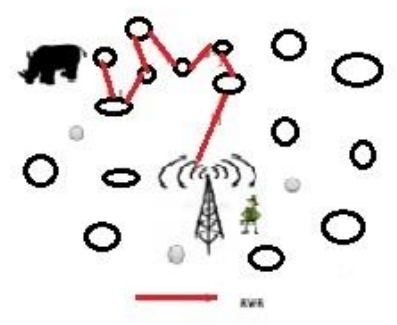

(b) RWR
Fig 2.1 (a-b) Random walking routing protocols.

Prasenjit Chanak et al., [4] proposed a fault tolerant routing which involves fault recovery process with fault detection scheme, referred to as energy efficient fault tolerant multipath routing scheme for wireless sensor network (EEFTMR).

Occurrences of fault in wireless sensor network are broadly classified in two groups; transmission fault and node fault. The node fault is further classified into five groups. These are power fault, sensor circuit fault, microcontroller fault, transmitter circuit fault and receive circuit fault. Energy efficiency is a prime metric in WSN performance analysis. This has motivated to propose this current algorithm for fault tolerant and energy efficient routing. In EEFTMR technique every sensor node transmits its data to BS through shortest path. If data and node fault occurs in the network, these are recovered very fast and data are transmitted to BS with minimum time and energy loss. The EEFTMR also controls the data traffic when data are transmitted to cluster head $(\mathrm{CH})$ or base station (BS).

\section{Fault tolerance data routing model}

In EEFTMR technique, when data are reaching to the neighboring destination node via data routing path, they first check their received data and their own sensed data. If these two are same then neighbor nodes are not forwarding the received data to others. If a node receives different data then receiver node sends receiving data toward the $\mathrm{CH}$ with shortest path. In EEFTMR technique, clusters head and BS are also connected to each other with the help of multiple data path. The shortest path is mainly used for fast energy efficient data routing towards BS. Other two backup paths are used for duplicate data routing, which makes the network path fault tolerant.

\section{Fault detection in EEFTMR}

In EEFTMR technique when a data is received by the destination node, they compare the received data with its own sensed data. If the compared value is less than the threshold value, then the sensor circuit of the node is good. If a sensor node receives data from neighbor nodes, then the sensor node receiver circuit is considered as good. If a sensor node has not received any data from its neighbor nodes for a long period of time then they send a message to other neighbor nodes to check the health of the neighbors. If all the neighbor nodes reply, then the sending node consider that there is some transmission fault occurring between communicating neighbors. However, if any neighbor node does not respond then the node confirm that its receiver circuit is faulty. On the other hand if a communication node is not responding then it will be declared as dead node and that has been informed to all the neighboring nodes.

\section{Fault recovery in EEFTMR}

Depending on the hardware condition of the node they are categorize as: normal node, traffic node, end node, dead node. The categorization helps improving the network lifetime and decreases the percentage of dead node in the network.

Ren-Cheng Jin et al.,[5] proposed a Passive cluster-based multipath routing protocol for wireless sensor networks. Energy efficiency and quality of service (QoS) are both essential issues in the applications of wireless sensor networks (WSNs) all along, which are mainly reflected in the development of routing and MAC protocols. However, there is little design for achieving the dual performances simultaneously. In this proposed approach, a practical passive clusterbased node-disjoint many to one multipath 
routing protocol has been designed to satisfy the requirements of energy efficiency and QoS in practical WSNs. Passive clustering approach is put to use in the first round, while active clustering technique is taken in the other rounds. The protocol includes two parts-passive clustering algorithm and multipath routing algorithm. As soon as an event occurs in the monitoring region, the protocol organized the entire network into clusters in terms of the passive clustering algorithm. After that, multiple paths are established among cluster heads. The data, which have been collected from member nodes by the cluster heads, are transmitted to the base station along multiple paths in the way of random linear network coding.

In Multipath routing algorithm (CBMP), searching multiple paths from multiple source nodes to one destination simultaneously is the main task. Instead of finding multiple paths between a specific source and a specific destination, the proposed protocol takes advantage of flooding in a typical route discovery process and finds multiple nodedisjoint paths from every sensor node to the common destination simultaneously. Two broadcasting phases, branch aware flooding and multipath expansion, are the cores of the multipath routing discovery process.

\section{Performance Analysis}

They have been categorized as well as differentiated into types of clustering methods based on a set of attributes as mentioned below. Ref acronym for abbreviations'.

\section{CONCLUSION}

Unlike centralized system, wireless sensor network is subjected to a unique set of resource constraints such as finite on-board battery power, limited network bandwidth etc. Each sensor nodes operate un tethered and has small amount of memory for signal processing and task scheduling. For communication, the main consideration is that communication paths consisting of many short hops may be more energy efficient than paths using a few long hops. Keeping all these points in mind, the above literature survey has been done or presented to design an energy efficient way to conduct routing in wireless sensor networks in order to preserve scarce energy resource and increase the lifetime of the network. Cluster Based Energy Efficient Routing (CBER) is one such approach that is manages energy expenditure in a more efficient manner through cluster formation.

\section{REFERENCES}

[1] Ankit Thakkar; Ketan Kotecha (26 June 2014 ). Cluster Head Election for Energy and Delay Constraint Applications of Wireless Sensor Network. IEEE Sensors Journal. 14 (issue 8), 2658 - 2664.

[2] Sajid Hussain et al., School of Computer Science, Acadia University Wolfville, Nova Scotia, Canada. Hierarchical Cluster Based Routing.

[3] R Manjula, Raja Datta. (28 February 2014). An Energy-Efficient Routing Technique for Privacy Preservation of Assets Monitored with WSN. Students' Technology Symposium (TechSym), 2014 IEEE . 325-330.

[4] PrasenjitChanak, TuhinaSamanta , Indrajit Banerjee. (December 2013). Energy efficient fault-tolerant multipath routing scheme for wireless sensor networks. www.sciencedirect.com/science/journal/1005888 5. 20 (6), 42-48.

[5] In, Ren-Cheng; Gao, Teng; Song, JinYan; Zou, Ji-Yan; Wang, Li-Ding. (29 March 2013). Passive cluster-based multipath routing protocol for wireless sensor networks. Springer Science+Business Media New York. 19 (Issue 8, p1851), 1851-1866.

[6] Ali Norouzi and A. Halim Zaim.(16 February 2014). Genetic Algorithm Application in Optimization of Wirel.ess Sensor Networks. Hindawi Publishing Corporation and The Scientific World Journal. 2014(Article ID 286575), 1-15. 


\section{Acronym}

Ce: Centralized; Di: Distributed; Hy: hybrid;

Pr: Probabilistic; It: Iterative; De: Deterministic;

Ad: Adaptive; Ra: Random; Pa: Proactive; Re:

Reactive; DF: Data Aggregation/Fusion; LB:
Load Balancing; FT: Fault Tolerant; GC: Guarantee of Connectivity; LE: Lifetime Extension; QoS: Quality of Service; Misc: Others.

\begin{tabular}{|c|c|c|c|c|c|c|c|c|c|c|c|c|c|c|c|c|c|c|}
\hline \multirow[t]{2}{*}{ Protocol used } & \multicolumn{3}{|c|}{ Control Manners } & \multicolumn{2}{|c|}{\begin{tabular}{|l|} 
Execution \\
nature
\end{tabular}} & \multicolumn{3}{|c|}{$\begin{array}{l}\begin{array}{l}\text { Parameters for } \\
\text { CH election }\end{array} \\
\end{array}$} & \multicolumn{3}{|c|}{ Proactivity } & \multicolumn{7}{|c|}{ Objectives } \\
\hline & $\mathrm{Ce}$ & Di & Hy & Pr & It & De & Ad & $\mathbf{R a}$ & $\mathbf{P a}$ & $\mathbf{R e}$ & Hy & DF & LB & FT & GC & LE & QoS & Misc \\
\hline EDIT & & $\mathbf{X}$ & & & $\mathbf{X}$ & & $\mathbf{X}$ & & & $\mathbf{X}$ & & $\mathbf{X}$ & $\mathbf{X}$ & & & $\mathbf{X}$ & & $\mathbf{X}$ \\
\hline HCR & & $\mathbf{X}$ & $\mathbf{X}$ & $\mathbf{X}$ & & & $\mathbf{X}$ & & & $\mathbf{X}$ & & $\mathbf{X}$ & $\mathbf{X}$ & & & $\mathbf{X}$ & & $\mathbf{X}$ \\
\hline EPR & $\mathbf{X}$ & & & $\mathbf{X}$ & & $\mathbf{X}$ & & & & $\mathbf{X}$ & & $\mathbf{X}$ & $\mathbf{X}$ & & $\mathbf{X}$ & & $\mathbf{X}$ & $\mathbf{X}$ \\
\hline EEFTMR & & & & & & & & & & $\mathbf{X}$ & & $\mathbf{X}$ & $\mathbf{X}$ & $\mathbf{X}$ & $\mathbf{X}$ & $\mathbf{X}$ & $\mathbf{X}$ & $\mathbf{X}$ \\
\hline $\begin{array}{l}\text { Event-driven } \\
\text { CBMP }\end{array}$ & & $\mathbf{X}$ & & & $\mathbf{X}$ & $\mathbf{X}$ & $\mathbf{X}$ & & & $\mathbf{X}$ & & $\mathbf{X}$ & $\mathbf{X}$ & $\mathbf{X}$ & $\mathbf{X}$ & $\mathbf{X}$ & & $\mathbf{X}$ \\
\hline
\end{tabular}

